\title{
As expectativas de pais e profissionais de enfermagem em relação ao trabalho da enfermeira em UTIN
}

\author{
PARENTS AND NURSING STAFF'S EXPECTATIONS REGARDING THE NURSE'S WORK IN ANICU \\ LAS EXPECTATIVAS DE LOS PADRES Y PROFESIONALES DE ENFERMERÍA EN \\ RELACIÓN AL TRABAJO DE LAENFERMERA EN UCIN
}

Ivone Kamada', Semíramis Melani Melo Rocha²

1 Enfermeira.
Professora Adjunta -
Departamento de
Enfermagem -
Universidade de
Brasília.Doutora em
Enfermagem.
kamada@unb.br
2 Professora Titular -
Departamento de
Enfermagem
Materno-Infantil e
Saúde Pública -
Escola de Enferma-
gem de Ribeirão
Preto - Universidade
de São Paulo.
smmrocha@
eerp.usp.br

\section{RESUMO}

O objetivo geral foi identificar as expectativas dos pais e da equipe de enfermagem relativas ao trabalho da enfermeira pela ótica em Unidade de Terapia Intensiva Neonatal. Foi realizada uma pesquisa descritiva por meio de uma abordagem qualitativa com 30 entrevistas entre pais, enfermeiras, técnicas e auxiliares de enfermagem de uma Unidade de Terapia Intensiva Neonatal do interior do Estado de São Paulo. Os resultados demonstraram novas expectativas por parte de pais e profissionais em relação ao desempenho dos profissionais de enfermagem. Os conhecimentos evidenciados como necessários para a atuação da enfermagem foram: abordagem centrada na família, técnicas de relações interpessoais e diferenciação entre tecnologia e conhecimento científico. Concluiu-se ser necessário uma atuação mais incisiva da enfermeira no cuidado de enfermagem, adequando a utilização dos avanços tecnológicos com conhecimentos humanos, sobretudo nas relações interpessoais entre familiares e equipe; contemplando atividades de educação continuada, a exemplo de curso de especialização.

\section{DESCRITORES}

Papel do profissional de enfermagem.

Unidades de Terapia Intensiva

Neonatal.

Cuidados de enfermagem.

\section{ABSTRACT}

The general purpose of this investigation was to identify parent and nursing staff expectations regarding the nurse's role in Neonatal Intensive Care Units (NICU). A descriptive study was carried out using a qualitative approach and interviews were conducted at a NICU in the interior of the State of São Paulo. Results showed new expectations on the part of parents and professionals regarding the role of NICU nurses. The knowledge identified as necessary were a family-centered approach, interpersonal relations techniques, and differentiation between technology and scientific knowledge. The conclusion is that NICU nurses need to play a more incisive role in the nursing care process, adjusting the use of technological advances to human knowledge, particularly in the area of interpersonal relationships between family members and staff, which includes activities of continuing education, such as specialization courses.

\section{KEY WORDS}

Nurse's role.

Intensive Care Units, Neonatal. Nursing care.

\section{RESUMEN}

El objetivo general de esta investigación fue identificar las expectativas relacionadas al trabajo de la enfermera a partir de la óptica de los padres y del equipo de enfermería en Unidad de Cuidados Intensivos Neonatal. Realizamos una investigación descriptiva a partir de un enfoque cualitativo con 30 entrevistas a padres, enfermeras y técnicas de enfermería. Las entrevistas fueron realizadas en una Unidad de Cuidados Intensivos Neonatal, del interior del Estado de Sao Paulo. Los resultados demostraron las nuevas expectativas por parte de los padres y profesionales con relación al desempeño del profesional de enfermería. Los conocimientos necesarios evidenciados fueron: abordaje centrado en la familia, técnicas de relaciones interpersonales, diferenciación entre tecnología y conocimiento científico. Se con-cluye que es necesario una actuación más incisiva de la enfermera en el cuidado de enfermería, sobre todo en las relaciones interpersonales entre familiares y el equipo; contemplando actividades de educación contínua a ejemplo del curso de especialización.

\section{DESCRIPTORES}

Rol de la enfermera.

Unidades de Terapia Intensiva

Neonatal.

Atención de enfermería. 


\section{INTRODUÇÃO}

Esta investigação foi desenvolvida com o intuito de identificar novos desafios e expectativas que a evolução da tecnologia e dos cuidados intensivos têm trazido aos profissionais de enfermagem que trabalham em Unidade de Terapia Intensiva Neonatal (UTIN).

Numa revisão bibliográfica, verificamos que o conhecimento utilizado em UTIN pode ser apreendido nas seguintes temáticas: a humanização do trabalho em função da introdução de novas tecnologias; a participação da família no contexto da UTIN; o estresse dos pais e da equipe; o ambiente e seu efeito sobre o recém-nascido; a alimentação; a termorregulação; a assistência respiratória; a dor; a higiene; as questões éticas; a educação continuada para os profissionais e os modelos de assistência ${ }^{(1-9)}$. Há uma preocupação com cuidados personalizados, envolvendo os pais na assistência, individualizando cada criança, procurando relações humanas entre a equipe, os pais e o recém-nascido ${ }^{(1,7-8,10-11)}$.

Estudos mostram que o ambiente da UTIN pode interferir na maturação e organização do sistema nervoso central desses recém-nascidos. A rotina dos procedimentos e os altos ruídos resultam em significantes mudanças nas respostas comportamentais e fisiológicas do recémnascido, como o atraso no desenvolvimento cognitivo, emocional, físico, neurológico e sensitivo. As intervenções de enfermagem devem ser direcionadas para ajudar na transição da vida intra-uterina para a extra-uterina, mantendo um ótimo desenvolvimento, prevenindo a estimulação indesejada e o estresse, o que resulta em significantes mudanças nas respostas comportamentais e fisiológicas do recém-nascido ${ }^{(3-4,12-15)}$.

As intervenções de enfermagem devem ser direcionadas para ajudar na transição da vida intra-uterina para a extra-uterina, mantendo um ótimo desenvolvimento, prevenindo a estimulação indesejada e 0 estresse. balmente as expectativas, uns em relação aos outros, para ue possamos entender, nas relações intersubjetivas, a apli-

Para tanto, temos como objetivo geral deste estudo, identificar expectativas relativas ao trabalho da enfermeira a partir da ótica dos pais e da equipe de enfermagem em Unidade derapia Intensiva Neonatal.

Os objetivos específicos são: 1) Verificar as expectativas dos pais de neonatos internados em UTIN sobre a assistência de enfermagem; 2) Verificar quais os conhecimentos que a enfermeira que trabalha em UTIN identifica como necessários para o seu desempenho e 3) Verificar, entre os demais membros da equipe de enfermagem, quais as expectativas sobre o desempenho da enfermeira que trabalha na UTIN e sobre seu próprio desempenho.

\section{METODOLOGIA}

Tendo em vista os propósitos deste estudo, optamos por realizar pesquisa descritiva, a partir de uma abordagem qualitativa, utilizando como principal fonte de dados uma unidade de terapia intensiva neonatal, para esse estudo.

Existem autores $^{(17)}$ que referem como propósitos da pesquisa descritiva observar, descrever e explorar aspectos de uma situação, tendo como uma de suas vantagens o fato de que tende a ser bastante realista, sendo capaz de ampliar a nossa compreensão a cerca de temática a ser estudada.

A pesquisa descritiva utiliza recursos como registro, análise e o correlacionamento de fenômenos sem manipulá-los, tendo o ambiente natural como principal fonte de dados para descobrir a natureza e as características de um fenômeno ${ }^{(18)}$.

Neste estudo, partimos de uma coleta de dados empíricos primários, isto é, depoimentos. Procuramos construir uma reflexão sobre as expectativas relativas ao trabalho da enfermeira ouvindo as pessoas, tanto a equipe profissional, quanto os pais, em relação ao trabalho da enfermeira. Ao ouvi-los estamos apreendendo qual o significado do trabalho da enfermeira e o que ele representa para o conjunto das pessoas que interagem com ela no cuidado. Assim, temos a partir da visão, da percepção, na intersubjetividade dessas pessoas, uma relação dos conhecimentos necessários às enfermeiras no preparo para atuar numa UTIN.

Escolhemos a Unidade de Terapia Intensiva Neonatal de um grande centro de referência à saúde do interior do Estado de São Paulo, por ser um hospital de grande porte, alta tecnologia e ampla demanda. suas vivências cotidianas. É preciso que os sujeitos que operam o conhecimento técnico e científico manifestem ver- 
Para seu desenvolvimento, utilizamos como técnica de coleta de dados a entrevista. Os dados foram coletados individualmente, utilizando a técnica de entrevista semidiretiva ou semidirigida que não é inteiramente aberta nem encaminhada por um grande número de perguntas precisas. $\mathrm{O}$ investigador dispõe de perguntas-guia, relativamente abertas, as quais permitem que o entrevistado fale, estruturando o seu pensamento em torno do objeto perspectivado, mas reencaminhando a entrevista para os objetivos cada vez que o entrevistado se afaste deles ${ }^{(19-20)}$.

A participação na pesquisa foi voluntária e as entrevistas foram realizadas pela pesquisadora. O projeto foi previamente aprovado pelo Comitê de Ética em Pesquisa do referido hospital. Os pesquisados foram informados sobre os objetivos do estudo, a garantia do anonimato e o uso de gravador. Assim, as entrevistas foram gravadas e, posteriormente, transcritas na íntegra.

Elaboramos roteiros de entrevistas para as enfermeiras, para as auxiliares e técnicas de enfermagem e para os pais, todos com o mesmo conteúdo, mas com forma de abordagem diferenciada (ANEXOS I, II e III). As entrevistas foram realizadas num período de três meses, em local adequado, durante os plantões da manhã, tarde e noturno. Entrevistamos sete enfermeiras, duas técnicas e doze auxiliares de enfermagem, oito mães e um pai. Para chegar à compreensão ou ao significado das falas, a análise consistiu na leitura e releitura do material; apreensão da informação contida nas falas; atribuição de sentidos e significados específicos tomando por referência a condição do entrevistado e o contexto do objeto de pesquisa.

Para esta investigação, utilizamos a análise temática, na modalidade análise de avaliação que incide sobre os juízos formulados pelo entrevistado ${ }^{(20)}$. Desta forma, procuramos os diferentes juízos formulados pelo locutor, para atender ao objetivo de identificar as expectativas relativas ao trabalho da enfermeira a partir da ótica dos pais e da equipe de enfermagem em Unidade de Terapia Intensiva Neonatal. As entrevistas foram analisadas no seu todo, buscando o significado do que emergiu das falas em relação ao cenário onde as pessoas estavam inseridas.

$\mathrm{Na}$ análise dos dados, apresentamos algumas falas para ilustração, identificadas da seguinte forma:

- (M...) - mãe entrevista $\mathrm{n}^{\circ}$... Refere-se à identificação das entrevistas realizadas com as mães, sendo numeradas em ordem crescente de acordo com sua realização. A letra $M$ identifica a categoria da entrevistada, no caso, mãe;

- (Ef ...) - enfermeira entrevista no $\ldots .$. Refere-se à identificação das entrevistas realizadas com as enfermeiras, sendo numeradas em ordem crescente de acordo com sua realização. As letras Ef identificam a categoria das entrevistadas, no caso, enfermeiras;
- (TE ...) - técnicas de enfermagem entrevista no…,. Refere-se à identificação das entrevistas realizadas com as técnicas de enfermagem, sendo numeradas em ordem crescente de acordo com sua realização. As letras TE identificam a categoria das entrevistadas, no caso, técnicas de enfermagem;

- $(\mathrm{AE} . .$.$) - auxiliares de enfermagem entrevista no… Refe-$ re-se à identificação das entrevistas realizadas com as auxiliares de enfermagem, sendo numeradas em ordem crescente de acordo com sua realização. As letras AE identificam a categoria das entrevistadas, no caso, auxiliar de enfermagem.

A fala dos entrevistados foi mantida, na íntegra, sem muitas das convenções da língua padrão culta.

\section{RESULTADOS}

A análise dos dados será apresentada de acordo com os grupos de sujeitos: pais, enfermeira e equipe de enfermagem.

\section{As expectativas dos pais de neonatos sobre a assistência de enfermagem}

Quando perguntamos às mães e ao pai como eles estavam vivenciando a internação do seu bebê em uma UTIN, a primeira afirmação foi sobre a dificuldade e o estresse que estavam sentindo. Esta afirmação não foi uma queixa sobre a assistência, mas sobre a natureza do problema. Ter um filho nascido prematuramente ou com alguma patologia leva os pais a se sentirem angustiados e impotentes.

Está sendo muito difícil (M1).

...esses 3 meses e 15 dias, acho que para toda mãe é estressante (M8).

Quando indagados sobre a atuação da enfermeira, os pais referem pouco contato com ela. Mal conseguem diferenciá-la dos demais agentes da equipe de enfermagem. Em contrapartida, identificam com precisão o médico responsável, que lhes transmite as informações e orientações. Além disso, identificam todos os médicos que circulam pela UTIN. Uma das mães afirmou que identifica a enfermeira pelo emblema que usa na roupa. Contudo, relatou que sua relação com as enfermeiras era muito distante. Não conseguiu caracterizar o papel da enfermeira na equipe.

A médica eu sei quem é! Mas essa ... enfermeira é meio difícil né? Porque elas todas usam branco (M1).

A enfermeira e a auxiliar para mim parece ser uma só (M2).

Para atender à demanda e controlar as atividades, a enfermeira assume a função de gerenciamento distanciandose dos cuidados diretos à criança. Alguns autores lembram que a introdução de equipamentos sofisticados no cuidado requer maior atenção da enfermeira, interpondo-se entre ela e seus pacientes ${ }^{(21)}$. 
A dificuldade em identificar as enfermeiras pode ser explicada, em parte, porque muitas vezes há uma fragmentação de responsabilidades, cada um trabalha de forma independente dos demais. As enfermeiras da UTIN encaminham as mães à outro setor para receberem orientações sobre ordenha, alimentação do bebê e cuidados pós-alta. Quando recebem alta da UTIN, os bebês são transportados para esse setor do hospital. Assim, a alta é preparada por outra equipe de enfermagem e as mães não têm oportunidade de um contato mais pessoal com a enfermeira durante a estadia de seu bebê na UTIN.

As mães relataram que recebem as informações da enfermeira da UTIN por ocasião da primeira visita, quando são orientadas sobre alguns procedimentos de rotina para permanecer na Unidade, como lavar as mãos, vestir avental, os horários de visitas para o restante dos familiares, não mexer nos aparelhos, etc.

Quando a mãe chega pela primeira vez na UTIN para ver seu filho, rodeado de aparelhos, é feito um preparo pela equipe profissional, muito valorizado pelas mães. Segundo elas, essas orientações diminuem sua ansiedade e as acalma. Este tipo de orientação não se mostrou muito significativo para as mães e elas não reconhecem quem faz estas orientações como a pessoa que coordena toda a equipe de enfermagem. Nota-se uma preocupação da enfermeira voltada para o cumprimento de normas e regras da instituição e a ausência de técnicas de relacionamento interpessoal para colocar os pais em situação mais confortável.

Os cuidados dispensados à criança, como higiene corporal, troca de fraldas, higiene do ambiente são percebidos pelos pais como função da enfermagem. Contudo, não diferenciam a atuação da auxiliar de enfermagem da atuação da enfermeira.

Uma atividade identificada como função da enfermeira é regular o aparelhinho. Na percepção dos pais, quem controla os aparelhos eletrônicos são enfermeiras e quem faz a limpeza da unidade são auxiliares. Pelas afirmações, inferimos que as enfermeiras não se identificam ao fazer as orientações ou quando se comunicam com os pais.

Se deu um problema no aparelhinho, vem uma enfermeira já olha para ver o que é, e quem está limpando a caminha dele é a auxiliar (M2).

Os pais confiam na capacidade da equipe de enfermagem em cuidar do bebê e manusear os equipamentos. Sentem-se respeitados, pois suas dúvidas são esclarecidas, suas necessidades são atendidas. Afirmam que o cuidado dispensado pela equipe de enfermagem aos seus bebês é muito carinhoso. Consideram a equipe de enfermagem dedicada ao trabalho, atenciosa com os bebês e com os pais, competente e responsável. Muitas vezes, eles observam o que estão fazendo não só com o seu filho, mas também com as outras crianças que estão na mesma enfermaria ou até em outras.
Percebemos nas falas dos pais, que a equipe de enfermagem da UTIN estabelece um vínculo com os recém-nascidos. A equipe de enfermagem sente que o trabalho do cuidar exige grande dedicação e que, o vínculo com os pacientes é tão prazeroso que garante a permanência dos cuidadores numa área de trabalho tão difícil ${ }^{(22)}$.

\section{É o gesto que elas cuidam não só do meu filho. É como se} fosse delas, é brincando. Poderia estar chorando, ela vai lá, brinca, como se fosse filho mesmo né? Saber porque que tá chorando, falam, você tá nervosinho?, isso vai passar (M2).

As mães consideram o trabalho médico muito importante, pois são eles que prescrevem as condutas a serem tomadas na assistência da criança, mas o trabalho da enfermagem é fundamental. Saber quem são as enfermeiras parece não ter muito significado para as mães, uma vez que dificilmente recorrem a elas para os questionamentos relativos ao tratamento Elas reconhecem os cuidados de enfermagem, entretanto não conseguem defini-los. Apesar de não diferenciarem a enfermeira dos demais membros da equipe, identificam a assistência de enfermagem como um todo e valorizam positivamente $o$ atendimento que recebem.

Na saúde, o objeto de trabalho é compartilhado por diferentes profissionais, que se articulam em torno de uma única finalidade, na qual o médico tem posição central na condução do processo terapêutico, mas, suas ações não podem dispensar a participação de outros profissionais ${ }^{(23)}$.

Para alguns pais, apesar da boa assistência que suas crianças recebem, a comunicação e o bom entendimento da equipe são fundamentais. Uma das dificuldades relatadas pelos pais foi a distância entre sua residência e o hospital. Alguns dependem de meios de transporte da empresa na qual trabalham para se locomover, pois não residem na mesma cidade. Assim, alguns suportes sociais são necessários para ajudá-los neste momento difícil. Estes suportes têm muitos significados, quando as condições econômicas são mais precárias e eles não dispõem de recursos próprios.

As mães demonstram pouca curiosidade sobre os aparelhos eletrônicos que circundam o bebê. Prestam atenção se o bebê está aquecido e recebendo cuidados como higiene, pesagem, troca de venóclise e se os profissionais cuidam do bebê com delicadeza e carinho. Algumas vezes, notam que luzes vermelhas se acendem. Então fazem perguntas e afirmam que ficam satisfeitas com as respostas. A venóclise tem mais significado para os pais do que os aparelhos. Ficam ansiosos para saber quando os médicos irão suspendê-lo. Muitas vezes, consideram que as punções frequientes são muito dolorosas e mal podem pegar a criança no colo ou mesmo manipulá-la com medo de perderem o acesso venoso e necessitar de novas punções. 
O fato de não poder pegar o bebê no colo é aceito pela maioria das mães, devido à instalação de aparelhos para controlar a termorregulação, a respiração e a alimentação e também pela punção venosa. Consideram que o tratamento é cuidadoso e necessário. Aguardam, com paciência, a melhora da saúde de seu bebê.

Com a vivência na UTIN, as mães vão adquirindo o vocabulário específico de terapias intensivas. Não sabemos se este vocabulário reflete a compreensão da mãe sobre a gravidade do caso e o risco ao qual seu bebê está submetido. Aparentemente, vão incorporando este vocabulário ouvindo as discussões durante a visita médica, ao mesmo tempo em que vão se adaptando emocionalmente ao cotidiano na UTIN.

Hoje ela está no CPAP, ela foi entubada várias vezes e extubada. Porque abaixam a saturação várias vezes, então chegam até a "ambuzar". Entuba e extuba, mas está no CPAP e por enquanto está reagindo bem (M4).

Os pais reconhecem no trabalho da enfermagem o cuidado que dedicam ao seu bebê. Para eles, o importante é manter os bebês limpos, medicados e cuidarem com delicadeza. Também atribuem significado à forma como a enfermagem trata os pais, muitas vezes, diminuindo sua ansiedade. Querem ser respeitados e reconhecidos. A comunicação com a equipe é fundamental. Saber quem são as enfermeiras parece não ter muito significado para as mães, uma vez que dificilmente recorrem a elas para questões que consideram fundamentais, mas identificam bem o médico responsável por seu filho.

\section{Conhecimentos necessários para o desempenho das enfermeiras de UTIN: visão das enfermeiras}

As enfermeiras da UTIN relataram não possuir especialização específica na área de cuidados intensivos neonatais, mas sentem a necessidade de atualização e de um aprofundamento. Assim, participam de pequenos cursos que surgem relacionados à área de neonatologia e buscam novos conhecimentos por meio de literatura, trabalhando as rotinas da unidade e participando de congressos. A humanização, a promoção do aleitamento materno e a reciclagem do pessoal são alguns aspectos que as enfermeiras consideram importantes a serem buscados.

\footnotetext{
... mas a busca do conhecimento tem que ser contínua, e isso que é importante (Ef4).

... a gente lê bastante né? Se você tem interesse acaba lendo. No começo eu lembro que eu procurava muito porque eu gostava, mas eu acho que ainda há falhas, deveria ter mais aulas direcionadas mesmo (Ef2).
}

A introdução de equipamentos tecnológicos na unidade é feita pelos docentes da medicina que, muitas vezes, trazem experiência de outros países. Quando esses equipamentos são adquiridos para a unidade, a orientação do uso é feita pelos técnicos da empresa vendedora, em uma aula, mas as enfermeiras acham que essa orientação não é suficiente. Segundo as entrevistadas, a introdução de novos equipamentos deveria ser mais discutida, pois, quando o aparelho está em uso, surgem alguns problemas que não conseguem resolver.

As enfermeiras consideram que a busca de novas tecnologias diminui a humanização da assistência, pois a tecnologia envolve o uso de equipamentos que algumas vezes substituem o trabalho manual, distanciando o contato direto com a criança. Dessa forma, a "humanização" do cuidado, como preconizado na literatura tem sido pouco trabalhada, na opinião das entrevistadas. O contato físico com a criança e o calor humano são muito importantes para o seu desenvolvimento. Deve haver um vínculo maior da enfermagem com a mãe e com o recém-nascido. Esse tipo de cuidado é preconizado no curso de graduação, e reforçado nos estágios, na assistência a recém-nascidos maiores. Em UTIN, as crianças internadas são muito pequenas e estão sempre em incubadora ou berço de terapia intensiva. $\mathrm{O}$ contato físico entre as enfermeiras e a criança fica prejudicado. Como o número de enfermeiras é reduzido e as atividades são bastante complexas, a falta de tempo é considerada um empecilho para a realização de uma assistência mais humanizada e para manter contato mais personalizado com a mãe.

... a tecnologia afasta um pouco da humanização, eu acho
que as duas estão... pelo menos o que eu sinto aqui é que
não está interligada não, às vezes a busca de novas
tecnologias diminui a humanização (Ef1).
... você ter que lidar mais com o ser né? Com a pessoa, com
a mãe, com a criança do que com a parte tecnológica (Ef3).

Enquanto lamentam o distanciamento que a tecnologia acarreta, as enfermeiras reconhecem nela um fator que facilita muito o seu trabalho. Trabalhar com tecnologia de ponta significa a sobrevivência de recém-nascidos de alto risco, com o uso de equipamentos cada vez mais complexos, mas isso não substitui o trabalho da enfermeira. Há momentos em que pode haver falha do equipamento e, também, falta de pessoal para controlá-lo.

O processo de enfermagem é visto como muito importante na assistência, pois ele ajuda a enfermeira a organizar, sistematizar e conceituar a prática de enfermagem, assim, a criança é vista como um todo. Também permite aos enfermeiros diferenciar sua prática daquelas dos médicos e de outros profissionais de saúde. A estrutura do processo de enfermagem envolve as etapas de histórico, diagnóstico de enfermagem, prescrição, implementação e evolução. Cada etapa é essencial na resolução do problema estando inter-relacionada com as outras etapas ${ }^{(24)}$. Atualmente, as enfermeiras estão iniciando sua implantação na UTIN com algumas dificuldades pelo reduzido número de enfermeiras. 
Muitas enfermeiras percebem que a realização de um curso de especialização para atuar na UTIN é um fator fundamental para a assistência. Algumas relatam que têm incentivos da chefia de enfermagem para estarem participando de cursos em busca de novos conhecimentos, com flexibilização nas escalas, mas têm dificuldades econômicas. Por isto, argumentam que a administração do hospital deveria programar a especialização das enfermeiras para atuar em UTIN.

\begin{abstract}
Eu também acho importante a especialização, porque apesar do conhecimento que a gente tem aqui, ele atualiza mais a gente, em termos de conceitos. Então, se a gente não tiver curiosidade e buscar, a gente não vai saber porque está fazendo, então eu acho importante a gente estar se aprofundando ... (Ef3).
\end{abstract}

Em síntese, apesar das enfermeiras, reconhecerem os avanços tecnológicos como responsáveis pela melhoria da qualidade de vida dos recém-nascidos, elas atribuem a esses avanços certo distanciamento, que se interpõem entre elas, as crianças e as mães. A falta de tempo justifica o fato de não estarem disponíveis para cercarem os pais de mais atenção e cuidado.

\section{As expectativas sobre o desempenho da enfermeira e dos membros da equipe de enfermagem da UTIN: a visão dos auxiliares e técnicos de enfermagem.}

Algumas auxiliares vêem o trabalho na UTIN como estressante e difícil pela falta de material e pessoal. Não há um preparo psicológico, providenciado pela administração do hospital, a fim de prepará-las para lidar com as situações de estresse.

... a gente sente muito, é a falta de apoio psicológico, porque sofremos uma pressão e um estresse muito grande, e muitas vezes você sente que não deu conta, não sei, está faltando alguma coisa (AE7).

Os argumentos utilizados para justificar essa carência são o reduzido número de funcionárias, as condições em que se encontram as crianças e a necessidade de manter a equipe no próprio serviço cotidianamente, sem tempo para educação continuada. Assim, a administração de recursos humanos da instituição não encontra formas para aprimorar o trabalho da equipe de enfermagem. Às vezes, a função da enfermeira é vista por algumas auxiliares com certo incômodo, pois, na unidade, alguns procedimentos são executados somente pela enfermeira.

Há na enfermagem uma divisão de trabalho, na qual as técnicas e auxiliares de enfermagem estão subordinadas à autoridade da enfermeira. Cabe à enfermeira as funções mais intelectuais de supervisão e controle e às técnicas e auxiliares o trabalho mais manual ${ }^{(25)}$. Além desta divisão, há uma seleção de cuidados atribuídos a diferentes profissionais, de forma hierarquizada, de acordo com sua complexidade. As técnicas mais invasivas são atribuídas a profissionais mais qualificados. A presença da enfermeira sempre é esperada nas ur- gências e intercorrências durante o plantão, pois as auxiliares e técnicas sentem-se mais seguras durante o processo.

A introdução de tecnologia no berçário é vista como uma dificuldade, pois os manuais de orientação são escritos em inglês e elas não dominam este idioma. Então, quando surgem dúvidas, perguntam para as enfermeiras. Lidar com incubadoras é considerado mais fácil do que com os respiradores. As técnicas e auxiliares sentem a necessidade de um treinamento mais específico para lidar com esses aparelhos.

As pessoas que trabalham há mais tempo na UTIN percebem que houve uma evolução da assistência às crianças de risco e que as enfermeiras têm participado de muitos cursos, enriquecendo o conhecimento que é passado para a equipe. Apesar dessa evolução, a chamada "humanização" da assistência é vista pelas auxiliares e técnicas como uma situação pouco valorizada pelos médicos; as mães ficam à espera de informações sobre o filho e nem sempre são atendidas, pois os médicos sempre dizem estar muito ocupados.

\section{... às vezes, tem mãe que procura informação, o médico naquela hora não pode dar, acho que eles deviam dar mais atenção (TE4).}

Para que as mães não saiam da UTIN sem informação, as auxiliares acabam chamando a enfermeira para orientá-las e tentar tranqüilizá-las. A interação entre as mães, os filhos e a equipe de enfermagem é vista com bons olhos pelas auxiliares e técnicas. Para elas, a mãe é respeitada na unidade e bem tratada. Algumas auxiliares estimulam as mães a pegarem o filho no colo e tocá-lo. Como a maioria das crianças permanece na UTIN durante um período prolongado, muitas vezes estabelece-se um vínculo afetivo entre a equipe, mãe e a criança, o que é gratificante para o pessoal que lidou o tempo todo com a criança.

O trabalho da enfermeira é visto pelas auxiliares e técnicas como importante na manutenção do bom estado da criança.

\section{CONSIDERAÇÕES FINAIS}

Verificou-se que os pais valorizaram o cuidado de enfermagem dispensado aos seus bebês. Referiram que os bebês são cuidados com delicadeza, segurança e que a equipe da UTIN é atenciosa e competente, demonstrando confiança, o que as acalmava. As mães relataram que não conseguem identificar a enfermeira entre as diferentes categorias de enfermagem. Por outro lado, identificam o médico com facilidade, pois é sempre o mesmo que atende seus filhos e passa as informações que desejam saber. Assim, percebemos que há uma necessidade das enfermeiras se identificarem melhor, serem mais incisivas na forma de falar com os pais, explicando que seu trabalho é coordenar e administrar a assistência de enfermagem em UTIN. É necessário preparar as enfermeiras para interagirem com os pais, bem como, conhecer a família como um todo, pois o cuidado integral inclui a família como objeto do cuidado. 
As enfermeiras que atuam na UTIN relataram não possuir especialização na área e que há uma necessidade de aprofundamento do conhecimento específico para lidar em uma unidade de terapia intensiva neonatal. Consideram que as orientações oferecidas em treinamentos e as informações dadas em cursos com técnicos dos aparelhos são insuficientes.

Verificamos que as enfermeiras consideram os avanços tecnológicos importantes para o cuidado ao recém-nascido. Eles são responsáveis pela sobrevida de crianças de alto risco e melhor qualidade de vida. Entretanto, contrapõem a tecnologia à "humanização" da assistência. Esta contraposição não é necessária, pois é possível conciliar tecnologia com humanização. Os conhecimentos sobre apego mãe-filho, relações interpessoais, a abordagem enfermagem da família são relativamente novos na formação da enfermeira. Estes conhecimentos requerem técnicas apropriadas para o cuidado. Sua implementação é necessária no curso de graduação em enfermagem e nos cursos de especialização em enfermagem pediátrica e neonatal. É também imprescindível para a formação de especialista em UTIN.

Para algumas auxiliares e técnicas o trabalho na UTIN é considerado estressante pelas condições de espaço físico, material, falta de compreensão da enfermeira em alguns momentos e principalmente pela falta de pessoal, pelo número reduzido de enfermeiras no plantão. A falta de experiência dificulta o bom andamento da assistência. Consideram o trabalho da enfermeira importante na orientação dos pais, o que não foi reconhecido pelas mães entrevistadas.

Por meio de metodologias de assistência apropriadas, que incluem fundamentação teórica da área de conhecimentos humanos, bem como, de inovações tecnológicas, é possível preparar enfermeiras que conciliem o uso de equipamentos complexos com relações interpessoais afetivas. É necessário trazer à compreensão que dinâmicas de relacionamento são também inovações tecnológicas. Desta forma, pode-se resolver essa dicotomia entre tecnologia e o que elas chamam de humanização.

\section{REFERÊNCIAS}

(1) Saunders AN. Changing nurses' attitudes toward parenting in the NICU. Pediatr Nurs. 1994;20(4):392-4.

(2) deLestard K, Lennox K. Developmental care: making your NICU a gentler place. Can Nurse. 1995;91(2):23-6.

(3) Symon A, Cunningham S. Handling premature neonates: a study using time-lapse video. Nurs Times. 1995;91(17):35-7.

(4) Zahr LK, Balian S. Responses of premature infants to routine nursing interventions and noise in the NICU. Nurs Res. 1995;44(3):179-85.
Não há cursos de educação continuada, providenciado pela administração do hospital a fim de prepará-las para lidar com as situações de estresse. Os argumentos utilizados para justificar essa carência são: o reduzido número de funcionárias, as condições em que se encontram as crianças e a necessidade de manter os funcionários continuamente no próprio serviço. Assim, a administração de recursos humanos não encontra formas de aprimoramento para a equipe de enfermagem. Apesar disto, as enfermeiras estão constantemente buscando novos conhecimentos. A tecnologia propiciou um avanço no processo terapêutico, reconhecido tanto pelos pais, como por auxiliares e técnicas de enfermagem e enfermeiras.

É preciso, também, que a administração do hospital reconheça seu papel de providenciar e manter todos os recursos necessários: equipamento, pessoal, treinamento, entre outros, porque, sendo uma unidade de cuidado intensivo, lida com situações de risco. É importante a interação entre a administração e a universidade, de tal forma que haja reciprocidade entre o conhecimento necessário para o desempenho no cuidado intensivo e os novos conhecimentos produzidos.

Um aspecto evidenciado nesse trabalho que resume a nosso ver, as expectativas referentes ao trabalho do profissional enfermeiro, na visão dos mesmos, é necessidade de um aprofundamento do conhecimento na área terapia intensiva neonatal.

Consideramos importante um curso de especialização para atuação numa UTIN, pois nesta investigação verificamos que as enfermeiras sentem necessidade de conhecerem os equipamentos e aplicar a sistematização da assistência de enfermagem como um instrumento que auxilie na qualidade da assistência. Este preparo formal poderá auxiliar a identificação da enfermeira em relação aos demais profissionais. Esses cursos de especialização devem abordar fisiopatologia, técnicas de acesso endovenoso, manuseio de equipamentos eletrônicos, banco de dados eletrônicos, técnicas de relações interpessoais para saber como trabalhar com emoções e, principalmente, o estresse.

(5) Bell RP, McGrath JM. Implementing a research-based kangaroo care program in the NICU. Nurs Clin North Am. 1996; 31(2):387-403.

(6) Als H, Duffy FH, McAnulty GB. Effectiveness of individualized neurodevelopmental care in the newborn intensive care unit (NICU). Acta Paediatr. Suppl. 1996;416:21-30.

(7) Mcgrath JM, Conliffe-Torres S. Integrating family-centered developmental assessment and intervention into routine care in the Neonatal Intensive Care Unit. Nurs Clin North Am. 1996;31(2):367-86. 
(8) Griffin T, Kavaugh K, Soto CF, White M. Parental evaluation of a tour of the Neonatal Intensive Care Unit during a high-risk pregnancy. J Obstet Gynecol Neonatal Nurs. 1997;26(1):59-65.

(9) Gibbins AS, Mc Donald, Chapman JS. Holding On: parents' perceptions of premature infants' transfers. J Obstet Gynecol Neonatal Nurs. 1996;25(2):147-53.

(10) Drosten-Brooks F. Kangaroo care: skin-to-skin contact in the NICU. MCN Am J Matern Child Nurs. 1993;18(5):250-3.

(11) Griffin T, Wishba C, Kavanaugh K. Nursing interventions to reduce stress in parents of hospitalized preterm infants. J Pediatr Nurs. 1998;13(5):290-5

(12) White-Traut RC, Nelso MN, Burns K, Cunningham N. Environmental influences on the developing premature infant: theoretical issues and applications to practice. J Obstet Gynecol Neonatal Nurs. 1994;23(5):393-401.

(13) Klaus MH, Martin RJ, Fanaroff AA. O Ambiente físico. In: Klaus MH, Fanaroff AA. Alto risco em neonatologia. $4^{\mathrm{a}} \mathrm{ed}$ Rio de Janeiro: Guanabara Koogan; 1995. p. 86-97.

(14) Glass PO. Recém-nascido vulnerável e o ambiente na Unidade de Tratamento Intensivo Neonatal. In: Avery GB, Fletcher MA, Macdonald MG. Neonatologia: fisiopatologia e tratamento do recém-nascido. $4^{\mathrm{a}}$ ed. São Paulo: MEDSI; 1999. p. 79-96.

(15) Blackburn S. Environmental impact of the NICU on developmental outcomes. J Pediatr Nurs. 1998;13(5):279-89.

(16) Lefrak-Okikawa L, Lund CH. Prática de enfermagem na Unidade de Tratamento Intensivo Neonatal. In: Klaus MH, Fanaroff AA. Alto risco em neonatologia. $4^{\mathrm{a}} \mathrm{ed}$. Rio de Janeiro: Guanabara Koogan; 1995. p. 156-67.
(17) Polit DF, Hungler BP. Fundamentos de pesquisa em enfermagem. Trad. de Regina Machado Garcez. $3^{\mathrm{a}}$ ed. Porto Alegre: Artes Médicas; 1995.

(18) Cervo AL, Bervian PA. Metodologia científica. $4^{\mathrm{a}}$ ed. São Paulo: Makron Books; 1996.

(19) Ruquoy D. Situação de entrevista e estratégia do entrevistador. In: Albarello L, Digneffe F, Hiernaux JP, Maroy C, Ruquoy $\mathrm{D}$, Saint-Georges P. Práticas e métodos de investigação em ciências sociais. Lisboa: Gradiva; 1997. p. 84-116.

(20) Quivy R, Campenhoudt LV. Manual de investigação em ciências sociais. Lisboa: Gradiva; 1998.

(21) Sandelowski M. Troubling distinctions: a semiotics of the nursing/technology relationship. Nurs Inq. 1999; 6:198-207.

(22) Shimizu E, Ciampone MHT. Sofrimento e prazer no trabalho vivenciado pelas enfermeiras que trabalham em Unidades de Terapia Intensiva em um hospital escola. Rev Esc Enferm USP. 1999;33(1):95-106.

(23) Lima MADS. O trabalho da enfermagem na produção de cuidados de saúde no modelo clínico [tese]. Ribeirão Preto: Escola de Enfermagem de Ribeirão Preto/USP; 1998.

(24) Potter PA, Perry AG. Fundamentos de enfermagem: conceitos, processo e prática. Rio de Janeiro; Guanabara Koogan; 2000 .

(25) Gonçalves RBM. Medicina e história: raízes do trabalho médico [dissertação]. São Paulo: Faculdade de Medicina da USP; 1979.

\section{ANEXO I \\ Roteiro de Entrevista - Mães e/ou Pais}

* Como o senhor(a) tem vivenciado a internação de seu(a) filho(a)?

Pretendemos ouvir das mães e/ou pais, se eles identificam os diferentes profissionais que atuam na UTIN, como vivenciam a internação do(a) filho(a), qual o tipo de expectativa que eles têm em relação ao cuidado de enfermagem.

\section{ANEXO II}

\section{Roteiro de Entrevista - Enfermeiras}

* Como a senhora vivencia o seu trabalho na UTIN?

Vamos procurar verificar, como ela identifica o seu trabalho enquanto enfermeira numa UTIN, qual o conhecimento necessário para atuar nessa unidade, e como ela vê a nova tecnologia utilizada.

\section{ANEXO III}

\section{Roteiro de Entrevista - Auxiliares e Técnicas de Enfermagem}

* Como a senhora vivencia o seu trabalho na UTIN?

Vamos procurar verificar, como ela identifica o trabalho da enfermeira, se ela diferencia o trabalho dos diferentes profissionais de enfermagem e qual o conhecimento necessário para ministrar cuidados para recém-nascidos em UTIN. 